\title{
ON THE EXISTENCE OF ELECTRICAL NETWORKS
}

\author{
C. E. CLARK
}

This paper is concerned with the existence of electrical networks which satisfy certain preassigned conditions. These conditions have to do with the existence of circuits with preassigned resistances in common.

Consider a finite set of points in euclidean 3-space and a set of straight line segments joining pairs of these points. Furthermore, suppose that no two of the segments intersect at an interior point, but any number of segments may have a common end-point. Each of the segments is called a branch of the graph. With each branch of the graph let there be associated a non-negative real number called the resistance of the branch. The graph together with the resistances is an electrical network. A circuit of a network is a topological circle of the network together with an orientation of this circle.

Let two circuits $C_{i}$ and $C_{j}$ have the branches $b_{i j}^{p}, p=1,2, \cdots$, in common. Let $r_{i j}^{p}$ be the resistance of $b_{i j}^{p}$. Let $\bar{r}_{i j}^{p}=r_{i j}^{p}$ if the orientations of $C_{i}$ and $C_{i}$ agree along $b_{i j}^{p}$ while $\bar{r}_{i j}^{p}=-r_{i j}^{p}$ if the orientations are opposite. Then $I_{i j}=\sum_{p} \bar{p}_{i j}^{p}, p=1,2, \cdots$, is the intersection of $C_{i}$ and $C_{j}$. We see that $I_{i i}$ is the sum of the resistances of the branches of $C_{i}$.

Let $C_{i}, i=1,2, \cdots, n$, be distinct circuits of a network. Then the matrix $\left\|I_{i j}\right\|, i, j=1, \cdots, n$, is the intersection matrix of the $C_{i}$. This matrix is symmetric and has non-negative diagonal elements. Any matrix with these two properties is an intersection matrix. An intersection matrix $M$ is realizable when there exists a network which has a set of circuits whose intersection matrix is $M$.

TheOREM 1. Given an intersection matrix

$$
\left\|I_{i j}\right\|, \quad i, j=1, \cdots, n \text {; }
$$

if $I_{i i} \geqq \sum_{j}\left|I_{i j}\right|, j \neq i, i=1, \cdots, n$, then (1) is realizable.

Proof. Choose $2 n$ points on each of $n$ oriented circles $S_{i}, i=1, \cdots, n$. These points divide each $S_{i}$ into $2 n$ branches. Denote the $2 n$ branches of $S_{i}$ by $b_{i 1}, o_{i 1}, b_{i 2}, o_{i 2}, \cdots, b_{i n}, o_{i n}$ with the $b$ 's separated by the $o^{\prime}$ 's. To each $o$ is assigned the resistance zero. We shall next identify $b_{i j}$ and $b_{j i}, i \neq j .{ }^{1}$ In the resulting figure there will be a circle that is the natural topological image of $S_{i}$. We shall say that this circle $i s S_{i}$.

${ }^{1}$ I.e., we bring the two end-points of $b_{i j}$ into coincidence with the end-points of $b_{j i}$ and replace these two branches by a single branch. To do this it may be necessary to replace any figure by its homeomorph. 
The identification of $b_{i j}$ and $b_{j i}$ can be performed in two ways. If $I_{i j}>0$, the identification is made so that $S_{i}$ and $S_{j}$ agree in orientation along their common branch; if $I_{i j}<0, S_{i}$ and $S_{j}$ have opposite orientations along their common branch; and if $I_{i j}=0$, we choose either identification. To the common branch is assigned the resistance $\left|I_{i j}\right|$.

To $b_{i i}, i=1, \cdots, n$, is assigned the resistance $I_{i i}-\sum_{j}\left|I_{i j}\right|, j \neq i$.

In the resulting network the $S_{i}, i=1, \cdots, n$, are circuits whose intersection matrix is (1).

THEOREM 2. If matrix (1) is realizable, and if a matrix (1a) is obtained from (1) by increasing a diagonal term $I_{i i}$ to $I_{i i}^{\prime}$, then (1a) is realizable.

Proof. Consider a realization of (1) and let $C_{i}$ be the circuit whose total resistance is $I_{i i}$. We break $C_{i}$ at one of its vertices $A$ and insert two branches $b_{i}$ and $b$ between the two ends $A_{1}$ and $A_{2}$ of the broken circuit. Any other branch that had been connected at $A$ may now be connected at either $A_{1}$ or $A_{2}$. To the branch $b_{i}$ is assigned the resistance $I_{i i}^{\prime}-I_{i i}$, while to $b$ is assigned the resistance zero. The new network gives a realization of (1a).

The above theorems show that if all except the diagonal elements of a symmetric matrix are given, then it is possible to assign the diagonal elements so that the resulting matrix is realizable. Also there are minimal sets of diagonal elements that can be assigned. But there is not a unique minimal set of diagonal elements as we now show by example.

We wish to find the smallest $a, b$, and $c$ so that

$$
\left\|\begin{array}{rrr}
a & 1 & 1 \\
1 & b & -1 \\
1 & -1 & c
\end{array}\right\|
$$

is realizable. We can take $a=1$. To do so we must have the second and third circuits $S_{2}$ and $S_{3}$ intersecting the first circuit $S_{1}$ along the same branch and both $S_{2}$ and $S_{3}$ agreeing in orientation with $S_{1}$ along this branch. Since $I_{23}=-1$, we must have $S_{2}$ and $S_{3}$ intersecting along another branch. This branch can have resistance 2 with $S_{2}$ and $S_{3}$ oppositely oriented here. This gives a realization of (2) with $a=1, b=3$, and $c=3$.

Also we can see that if $a<2$, then one other of the diagonal terms must be greater than 2 . Hence if $a=b=c=2$ makes (2) realizable, then this set of diagonal elements is a minimal set (in the sense that one element can not be decreased without increasing another). But 
by Theorem 1 the values $a=b=c=2$ make (2) realizable. Hence there is not. a unique minimal set of values for $a, b$, and $c$.

TheOREM 3. Given $I_{i j}, i \neq j$, of (1) (but not the diagonal elements), let $a$ be fixed; then we can take $I_{a a}=\max \left|I_{a j}\right|, j \neq a$, and find values for $I_{i i}, i \neq a$, so that (1) is realizable.

The proof is similar to the proofs of the preceding theorems.

Purdue University

\title{
ON THE SPHERICAL SURFACE OF SMALLEST RADIUS ENCLOSING A BOUNDED SUBSET OF n-DIMEN- SIONAL EUCLIDEAN SPACE ${ }^{1}$
}

\author{
L. M. BLUMENTHAL AND G. E. WAHLIN
}

1. Introduction. An $(n-1)$-dimensional spherical surface $S_{n-1, r}$ is the "surface" of an $n$-dimensional sphere of radius $r$ in $E_{n}$, the $n$-dimensional euclidean space. A given spherical surface encloses $M$, a subset of $E_{n}$, provided $M$ is contained in the sphere with this surface, while $M$ is enclosable by a given $S_{n-1, r}$ whenever $M$ is a subset of a sphere whose surface is congruent with $S_{n-1, r}$. The purpose of this article is to show (1) if $M$ is any bounded subset of $E_{n}$ (containing more than a single point) there exists a unique $S_{n-1, r}$ of smallest radius $r$ enclosing $M$ and (2) if $d$ is the diameter of $M$, then the radius of the unique smallest $S_{n-1, r}$ enclosing $M$ satisfies the relation $r \leqq[n / 2(n+1)]^{1 / 2} \cdot d$.

In a proof that abounds with algebraic difficulties, H. W. E. Jung established these results in his dissertation (1901) for the case of finite point sets and indicated their extension to infinite sets at the end of his long paper. ${ }^{2}$ Returning to the subject eight years later, Jung attempted a geometric proof for the case of $n$ points in a plane, but succeeded in obtaining in this later article only necessary conditions on the smallest circle enclosing a plane (finite) set, since his procedure yields the smallest circle only in case one is assured of the existence of such a circle. ${ }^{3}$ Though this fact can readily be supplied, the geometric considerations used by Jung are not easily extended to finite

\footnotetext{
${ }^{1}$ Presented to the Society, February 22, 1941.

${ }^{2}$ H. W. E. Jung, Ueber die kleinste Kugel, die eine räumliche Figur einschliesst, Journal für die reine und angewandte Mathematik, vol. 123 (1901), pp. 241-257.

${ }^{3} \mathrm{H}$. W. E. Jung, Ueber den kleinsten Kreis, der eine ebene Figur einschliesst, ibid., vol. 137 (1909), pp. 310-313.
} 